\title{
Some questions on quasinilpotent groups and related classes
}

\author{
M. J. Iranzo, J. Medina and F. Pérez-Monasor
}

\begin{abstract}
In this paper we will prove that if $G$ is a finite group, $X$ a subnormal subgroup of $X \mathrm{~F}^{*}(G)$ such that $X \mathrm{~F}^{*}(G)$ is quasinilpotent and $Y$ is a quasinilpotent subgroup of $\mathrm{N}_{G}(X)$, then $Y \mathrm{~F}^{*}\left(\mathrm{~N}_{G}(X)\right)$ is quasinilpotent if and only if $Y \mathrm{~F}^{*}(G)$ is quasinilpotent. Also we will obtain that $\mathrm{F}^{*}(G)$ controls its own fusion in $G$ if and only if $G=\mathrm{F}^{*}(G)$.
\end{abstract}

The generalized Fitting subgroup $\mathrm{F}^{*}(G)$ of a finite group $G$ is the product of the Fitting subgroup and the semisimple radical of $G$.

This generalized Fitting subgroup satisfies $\mathrm{C}_{G}\left(\mathrm{~F}^{*}(G)\right) \leq \mathrm{F}^{*}(G)$, for every finite group $G$. This property is similar to the corresponding one for the Fitting subgroup of a soluble group: $\mathrm{C}_{G}(\mathrm{~F}(G)) \leq \mathrm{F}(G)$. Quasinilpotent groups are those groups which coincide with their generalized Fitting subgroup. A group $G$ such that $\mathrm{F}^{*}(G)=\mathrm{F}(G)$ is a nilpotent-constrained group.

H. Bender stated that if $G$ is a nilpotent-constrained group, $X$ a subgroup of $G$ such that $X \mathrm{~F}(G)$ is nilpotent and $Y \leq \mathrm{N}_{G}(X)$, then $Y \mathrm{~F}\left(\mathrm{~N}_{G}(X)\right)$ is nilpotent if and only if $Y \mathrm{~F}(G)$ is nilpotent.

A well known theorem of Frobenius states that if a $p$-Sylow subgroup of $G$ controls its own fusion in $G$, then $G$ has a normal $p$-complement.

In this paper we will prove that if $G$ is a finite group, $X$ a subnormal subgroup of $X \mathrm{~F}^{*}(G)$ such that $X \mathrm{~F}^{*}(G)$ is quasinilpotent and $Y$ is a quasinilpotent subgroup of $\mathrm{N}_{G}(X)$, then $Y \mathrm{~F}^{*}\left(\mathrm{~N}_{G}(X)\right)$ is quasinilpotent if and only if $Y \mathrm{~F}^{*}(G)$ is quasinilpotent. Also we characterize when a nilpotent

2000 Mathematics Subject Classification: 20D10, 20F19.

Keywords: Nilpotent group, quasinilpotent group, injector, fusion. 
injector controls its own fusion in a nilpotent-constrained group or when a quasinilpotent injector controls its own fusion in a finite group.

Notations. All groups considered in this paper are assumed to be finite. The non-explicit notations are standard, see for instance [3]. We quote nevertheless the following:

$\mathfrak{N}$ : class of nilpotent groups,

S: class of soluble groups,

$\mathfrak{N}^{*}$ : class of quasinilpotent groups,

$\mathrm{F}(G)$ is the Fitting subgroup of $G$, i.e., the largest nilpotent normal subgroup of $G$.

If $\mathfrak{F}$ is a class of groups,

$\mathbf{s}_{n} \mathfrak{F}=\{G ; G \unlhd X$ for some $X \in \mathfrak{F}\}$,

$\mathrm{N}_{0} \mathfrak{F}=\left\{G ; G=\left\langle X_{1}, \ldots, X_{n}\right\rangle\right.$ for some $\left.X_{i} \unlhd \unlhd G, X_{i} \in \mathfrak{F}, 1 \leq i \leq n\right\}$,

A Fitting class $\mathfrak{F}$ is an $\mathbf{s}_{n^{-}}$and $\mathbf{N}_{0}$-closed class, that is, a class such that $\mathfrak{F}=\mathbf{s}_{n} \mathfrak{F}=\mathbf{N}_{0} \mathfrak{F}$.

If $\mathfrak{F}$ is a Fitting class, a subgroup $H$ of $G$ is an $\mathfrak{F}$-injector of $G$ whenever $H \cap N$ is an $\mathfrak{F}$-maximal subgroup of $N$, for every subnormal subgroup $N$ of $G$. We denote by $\operatorname{Inj}_{\mathfrak{F}}(G)$ the set of all $\mathfrak{F}$-injectors of $G$. The quasinilpotent injectors of a group $G$ are characterized as the maximal quasinilpotent subgroups containing the generalized Fitting subgroup of $G([3])$.

A group $G$ is said to be quasisimple if $G$ is perfect and $G / \mathrm{Z}(G)$ is simple.

The quasisimple subnormal subgroups of a (finite) group $G$ are called the components of $G$. The semisimple radical $\mathrm{E}(G)$ of $G$ is the join of its components.

We will need the description of some properties about the semisimple radical of a group. As we did not find any complete reference to it in the literature, for the sake of being selfcontained, we include the following:

Lemma 1 Let $G$ be a group. Then:

(1) If $\mathrm{F}^{*}(G) \leq H \leq G$ it follows that $\mathrm{E}(H)=\mathrm{E}(G)$.

(2) If $H$ is a subnormal subgroup of $G$ then $\mathrm{E}(H)$ is the product of all components $Q$ of $G$ such that $[Q, H] \neq 1$. In particular $\mathrm{E}(G) \leq \mathrm{N}_{G}(H)$.

(3) If $H \unlhd \unlhd H \mathrm{~F}^{*}(G)$, then $\mathrm{E}\left(\mathrm{N}_{G}(H)\right)=\mathrm{E}(G)$. 
Proof. (1) Clearly $\mathrm{E}(G) \leq \mathrm{E}(H)$. As $\mathrm{F}^{*}(G) \leq \mathrm{N}_{G}(\mathrm{E}(H))$ then $\mathrm{E}(H) \leq$ $\mathrm{E}(G)([2] 4.25)$, thus $\mathrm{E}(G)=\mathrm{E}(H)$.

(2) If $Q$ is a component of $G$ and $H \leq G$, then either $[Q, H]=1$ or $Q \leq[Q, H]$ ([7], X 13.18). When $H \unlhd \unlhd G$ the second alternative implies that $Q \leq H$. Therefore $\mathrm{E}(H)$ is the product of all components $Q$ of $G$ such that $[Q, H] \neq 1$. In consequence $\mathrm{E}(G) \leq \mathrm{N}_{G}(H)$.

(3) By $([2], 4.26) \mathrm{E}\left(\mathrm{N}_{G}(H)\right) \leq \mathrm{E}(G)$. On the other hand, by (1) and (2) we have that $\mathrm{E}\left(H \mathrm{~F}^{*}(G)\right)=\mathrm{E}(G) \leq \mathrm{N}_{G}(H)$, thus $\mathrm{E}(G) \leq \mathrm{E}\left(\mathrm{N}_{G}(H)\right)$. Therefore $\mathrm{E}(G)=\mathrm{E}\left(\mathrm{N}_{G}(H)\right)$.

In [8] we proved the following result:

Suppose that $N$ is a nilpotent normal subgroup of $G$ and let $X$ be a nilpotent subgroup of $G$ satisfying $\mathrm{C}_{G}(N \cap X) \leq X$. Then $N X$ is nilpotent.

As a consequence of this result, it is easy to obtain:

If $X$ is a subgroup of $\mathrm{F}(G)$ and $Y$ is a nilpotent subgroup of $\mathrm{N}_{G}(X)$ containing $\mathrm{F}\left(\mathrm{N}_{G}(X)\right)$, then $Y \mathrm{~F}(G)$ is nilpotent.

A generalization of this result would be:

If $X \mathrm{~F}(G)$ is a nilpotent subgroup of $G$ and $Y$ a subgroup of $\mathrm{N}_{G}(X)$ satisfying $Y \mathrm{~F}\left(\mathrm{~N}_{G}(X)\right)$ is nilpotent, then $Y \mathrm{~F}(G)$ is nilpotent.

In [1] $\mathrm{H}$. Bender had given an affirmative answer when $G$ is a nilpotentconstrained group. Next we will prove that this result is true without any restriction:

Proposition 2 Let $X \leq G$ with $X \mathrm{~F}(G)$ nilpotent and let $Y \leq \mathrm{N}_{G}(X)$ with $Y \mathrm{~F}\left(\mathrm{~N}_{G}(X)\right)$ nilpotent, then $Y \mathrm{~F}(G)$ is nilpotent.

Proof. Work by induction on the order of $G$.

If $R=\mathrm{F}(G) \mathrm{N}_{G}(X)<G$ then $X \mathrm{~F}(G) \unlhd R$ thus $X \mathrm{~F}(G) \leq \mathrm{F}(R)$ and $X \mathrm{~F}(R)=\mathrm{F}(R)$. Therefore, since $\mathrm{N}_{G}(X)=\mathrm{N}_{R}(X)$, by the inductive hypothesis, it follows that $Y \mathrm{~F}(R)$ is nilpotent, so $Y \mathrm{~F}(G)$ is nilpotent.

Thus we can suppose that $G=\mathrm{F}(G) \mathrm{N}_{G}(X)$, so $X \mathrm{~F}(G) \unlhd G$, then $X \leq$ $\mathrm{F}(G)$ and by the consequence of $([8], 2.2)$, it follows that $Y \mathrm{~F}\left(\mathrm{~N}_{G}(X)\right) \mathrm{F}(G)$ is nilpotent, so $Y \mathrm{~F}(G)$ is nilpotent. 
Proposition 3 Let $X$ be a quasinilpotent subgroup of $G$ satisfying $X \cap$ $\mathrm{F}^{*}(G) \unlhd \unlhd \mathrm{F}^{*}(G)$. If $\mathrm{C}_{\mathrm{F}^{*}(G)}\left(X \cap \mathrm{F}^{*}(G)\right) \leq X$ then $X \mathrm{~F}^{*}(G)$ is quasinilpotent.

Proof. Since $U=X \cap \mathrm{F}^{*}(G) \unlhd \unlhd \mathrm{F}^{*}(G)$ and $\mathrm{C}_{\mathrm{F}^{*}(G)}(U) \leq U$, by ([7], X 15.1) it follows that $U=\mathrm{E}(G)(U \cap \mathrm{F}(G))$ and $\mathrm{C}_{\mathrm{F}(G)}(U \cap \mathrm{F}(G)) \leq U$.

Then

$$
\begin{aligned}
\mathrm{C}_{\mathrm{F}(G)}(\mathrm{F}(X) \cap \mathrm{F}(G)) & =\mathrm{C}_{\mathrm{F}(G)}(X \cap \mathrm{F}(G))=\mathrm{C}_{\mathrm{F}(G)}(U \cap \mathrm{F}(G)) \leq U \cap \mathrm{F}(G) \\
& =\mathrm{F}(X) \cap \mathrm{F}(G) .
\end{aligned}
$$

Next, we will prove that $\mathrm{F}(X) \mathrm{F}(G)$ is nilpotent. It suffices to show that $\mathrm{F}(X) \mathrm{O}_{p}(G)$ is nilpotent for every prime $p$ in order of $\mathrm{F}(G)$. Consider the action of $\left(\mathrm{O}_{p}(G) \cap \mathrm{O}_{p}(X)\right) \times \mathrm{O}_{p^{\prime}}(\mathrm{F}(X))$ on $\mathrm{O}_{p}(G)$. Since

$$
\mathrm{C}_{\mathrm{O}_{p}(G)}\left(\mathrm{O}_{p}(G) \cap \mathrm{O}_{p}(X)\right) \leq \mathrm{C}_{\mathrm{F}(G)}(\mathrm{F}(G) \cap \mathrm{F}(X)) \leq \mathrm{F}(X),
$$

we have $\mathrm{C}_{\mathrm{O}_{p}(G)}\left(\mathrm{O}_{p}(G) \cap \mathrm{O}_{p}(X)\right) \leq \mathrm{O}_{p}(X)$ and $\mathrm{O}_{p^{\prime}}(\mathrm{F}(X))$ acts trivially on $\mathrm{C}_{\mathrm{O}_{p}(G)}\left(\mathrm{O}_{p}(G) \cap \mathrm{O}_{p}(X)\right)$. The Thompson's $P \times Q$-lemma implies that $\mathrm{O}_{p^{\prime}}(\mathrm{F}(X))$ also acts trivially on $\mathrm{O}_{p}(G)$. Then $\mathrm{F}(X) \mathrm{O}_{p}(G)$ is nilpotent.

On the other hand, since $\mathrm{E}(X)$ is a quasinilpotent perfect $U$-invariant subgroup, by $([7], \mathrm{X} 15.2)$, it follows that $\mathrm{E}(X) \unlhd \mathrm{E}(G)$ so $\mathrm{E}(X)=\mathrm{E}(G)$, then $X \mathrm{~F}^{*}(G)=\mathrm{E}(X)(\mathrm{F}(X) \mathrm{F}(G))$ that is quasinilpotent.

\section{Remarks.}

1. Notice that, as the following example shows, the condition of subnormality in the above result is necessary.

Let $G=\mathrm{GL}(2,5)$ and $Z=\mathrm{Z}(G)$. By ([6], II 7.3) there exists $X \leq G$, $X \cong \mathrm{C}_{24}$ satisfying $\mathrm{C}_{G}(X)=X$. If $D=X \cap \operatorname{SL}(2,5)$ then $|D|=6$ and if $\langle x\rangle \leq D$ such that o $(x) \not 4$ then by $\left([10]\right.$, page 163) $\mathrm{C}_{\mathrm{SL}(2,5)}(\langle x\rangle)=D$. Since $\mathrm{F}^{*}(G)=\mathrm{SL}(2,5) Z$, then:

$$
\begin{aligned}
\mathrm{C}_{\mathrm{F}^{*}(G)}\left(\mathrm{F}^{*}(G)\right. & \cap X)=Z \mathrm{C}_{\mathrm{SL}(2,5)}(\mathrm{SL}(2,5) Z \cap X) \\
& =Z \mathrm{C}_{\mathrm{SL}(2,5)}(\mathrm{SL}(2,5) \cap X) \leq Z \mathrm{C}_{\mathrm{SL}(2,5)}(\langle x\rangle)=Z D \leq X .
\end{aligned}
$$

As $|X \mathrm{SL}(2,5)|=|G|$, it follows that $G=X \mathrm{SL}(2,5)=X \mathrm{~F}^{*}(G)$, that is not quasinilpotent.

2. It is easy to prove that Proposition 3 is equivalent to the following:

Let $H \leq G$ such that $\mathrm{C}_{\mathrm{F}^{*}(G)}\left(H \cap \mathrm{F}^{*}(G)\right) \leq H$ and $H \cap$ $\mathrm{F}^{*}(G) \unlhd \unlhd \mathrm{F}^{*}(G)$. If $X$ is a quasinilpotent subgroup of $G$, such that $\mathrm{F}^{*}(H) \leq X \leq H$, then $X \mathrm{~F}^{*}(G)$ is quasinilpotent. 
Next we will obtain a version for quasinilpotent groups of $([12], 2.1)$.

Recall that if $N$ is a normal subgroup of $G$ and $\theta \in \operatorname{Irr}(N)$, then $\mathrm{I}_{G}(\theta)=$ $\left\{g \in G \mid \theta^{g}=\theta\right\}$ is the stabilizer of $\theta$ in $G$.

Corollary 4 Let $N$ be a quasinilpotent normal subgroup of $G$. Let $\theta \in$ $\operatorname{Irr}(N)$ and let $T=\mathrm{I}_{G}(\theta)$ the stabilizer of $\theta$ in $G$. If $T \cap \mathrm{F}^{*}(G) \unlhd \unlhd \mathrm{F}^{*}(G)$ and $X$ is a quasinilpotent subgroup of $G$ satisfying $\mathrm{F}^{*}(T) \leq X \leq T$ then $X \mathrm{~F}^{*}(G)$ is quasinilpotent.

Proof. Since $N \mathrm{C}_{G}(N) \leq T$ we have

$$
\mathrm{C}_{\mathrm{F}^{*}(G)}\left(\mathrm{F}^{*}(G) \cap T\right) \leq \mathrm{C}_{\mathrm{F}^{*}(G)}(N \cap T)=\mathrm{C}_{\mathrm{F}^{*}(G)}(N) \leq T .
$$

Now, by Remark 2, we obtain that $X \mathrm{~F}^{*}(G)$ is quasinilpotent.

Corollary 5 If $X \unlhd \unlhd \mathrm{F}^{*}(G)$ and $Y$ is a quasinilpotent subgroup satisfying $\mathrm{F}^{*}\left(\mathrm{~N}_{G}(X)\right) \leq Y \leq \mathrm{N}_{G}(X)$, then $Y \mathrm{~F}^{*}(G)$ is quasinilpotent.

Proof. Since $X \unlhd \unlhd \mathrm{F}^{*}(G)$, by Lemma 1 (3), it follows that $\mathrm{E}(G)=$ $\mathrm{E}\left(\mathrm{N}_{G}(X)\right)$, thus

$$
\begin{aligned}
\mathrm{N}_{G}(X) \cap \mathrm{F}^{*}(G) & =\mathrm{E}(G)\left(\mathrm{N}_{G}(X) \cap \mathrm{F}(G)\right) \leq \mathrm{E}(G) \mathrm{F}\left(\mathrm{N}_{G}(X)\right) \\
& =\mathrm{F}^{*}\left(\mathrm{~N}_{G}(X)\right) \leq Y .
\end{aligned}
$$

Hence,

$$
Y \cap \mathrm{F}^{*}(G)=\mathrm{N}_{G}(X) \cap \mathrm{F}^{*}(G) \unlhd \unlhd \mathrm{F}^{*}(G)
$$

Then

$$
\begin{aligned}
\mathrm{C}_{\mathrm{F}^{*}(G)}\left(Y \cap \mathrm{F}^{*}(G)\right) & =\mathrm{C}_{\mathrm{F}^{*}(G)}\left(\mathrm{N}_{G}(X) \cap \mathrm{F}^{*}(G)\right) \leq \mathrm{C}_{\mathrm{F}^{*}(G)}(X) \leq \mathrm{N}_{\mathrm{F}^{*}(G)}(X) \\
& =\mathrm{F}^{*}(G) \cap \mathrm{N}_{G}(X) \leq Y .
\end{aligned}
$$

Therefore, by Proposition 3, it follows that $Y \mathrm{~F}^{*}(G)$ is quasinilpotent.

The following example shows that, in the above result, the subnormality condition is necessary:

Example. Let $\Sigma_{7}=\mathrm{A}_{7}\langle(6,7)\rangle$ and $\mathrm{A}_{5} \leq \mathrm{A}_{7}=\mathrm{E}\left(\Sigma_{7}\right)=\mathrm{F}^{*}\left(\Sigma_{7}\right)$ (where $\mathrm{A}_{5}$ is considered as the group of all even permutations of the set $\{1,2,3,4,5\})$.

Clearly $\mathrm{N}_{\Sigma_{7}}\left(\mathrm{~A}_{5}\right)=\Sigma_{5}\langle(6,7)\rangle$ and $\mathrm{F}^{*}\left(\mathrm{~N}_{\Sigma_{7}}\left(\mathrm{~A}_{5}\right)\right)=\mathrm{A}_{5}\langle(6,7)\rangle$ however $\mathrm{F}^{*}\left(\mathrm{~N}_{\Sigma_{7}}\left(\mathrm{~A}_{5}\right)\right) \mathrm{F}^{*}\left(\Sigma_{7}\right)$ coincides with $\Sigma_{7}$, that is not quasinilpotent. 
Theorem 6 Let $X \unlhd \unlhd X \mathrm{~F}^{*}(G)$ where $X \mathrm{~F}^{*}(G)$ is quasinilpotent and let $Y$ be a quasinilpotent subgroup of $\mathrm{N}_{G}(X)$. Then $Y \mathrm{~F}^{*}\left(\mathrm{~N}_{G}(X)\right)$ is quasinilpotent if and only if $Y \mathrm{~F}^{*}(G)$ is quasinilpotent.

Proof. Suppose that $Y \mathrm{~F}^{*}\left(\mathrm{~N}_{G}(X)\right)$ is quasinilpotent. We argue by induction on $|G|$.

If $R=\mathrm{N}_{G}(X) \mathrm{F}^{*}(G)<G$, then $X \mathrm{~F}^{*}(G) \unlhd R$ and $X \mathrm{~F}^{*}(G) \leq \mathrm{F}^{*}(R)$, thus $X \unlhd \unlhd X \mathrm{~F}^{*}(G) \unlhd \mathrm{F}^{*}(R)$. Since $\mathrm{N}_{G}(X)=\mathrm{N}_{R}(X)$, by induction we obtain that $Y \mathrm{~F}^{*}(R)$ is quasinilpotent. Since $\mathrm{F}^{*}(G) \leq Y \mathrm{~F}^{*}(R)$, by Lemma 1 (1) we have $\mathrm{E}\left(Y \mathrm{~F}^{*}(R)\right)=\mathrm{E}(G)$, thus $Y \mathrm{~F}^{*}(G) / \mathrm{E}(G) \leq Y \mathrm{~F}^{*}(R) / \mathrm{E}(G)$ that is nilpotent so $Y \mathrm{~F}^{*}(G) \unlhd \unlhd Y \mathrm{~F}^{*}(R)$, then $Y \mathrm{~F}^{*}(G)$ is quasinilpotent.

Thus we can suppose that $G=\mathrm{N}_{G}(X) \mathrm{F}^{*}(G)$. Then $X \mathrm{~F}^{*}(G) \unlhd G$ and $X \unlhd \unlhd \mathrm{F}^{*}(G)$. Using Corollary 5 it follows that $Y \mathrm{~F}^{*}\left(\mathrm{~N}_{G}(X)\right) \mathrm{F}^{*}(G)$ is quasinilpotent. Since $\mathrm{E}\left(Y \mathrm{~F}^{*}\left(\mathrm{~N}_{G}(X)\right) \mathrm{F}^{*}(G)\right)=\mathrm{E}(G)$ we have $Y \mathrm{~F}^{*}(G)$ is a subnormal subgroup of $Y \mathrm{~F}^{*}\left(\mathrm{~N}_{G}(X)\right) \mathrm{F}^{*}(G)$ and $Y \mathrm{~F}^{*}(G)$ is quasinilpotent as desired.

Assume now that $Y \mathrm{~F}^{*}(G)$ is quasinilpotent. As $Y \mathrm{~F}^{*}(G) / \mathrm{E}(G)$ is nilpotent, then $Y \mathrm{E}(G)$ is a subnormal quasinilpotent subgroup of $Y \mathrm{~F}^{*}(G)$. Write $Y_{1}=Y \mathrm{E}(G)$. Then $Y_{1} \leq \mathrm{N}_{G}(X)$ by Lemma 1 (3). Notice that $\mathrm{F}(X), \mathrm{F}\left(Y_{1}\right)$, $\mathrm{F}\left(\mathrm{N}_{G}(X)\right)$ are subgroups of $C=\mathrm{C}_{G}(\mathrm{E}(G))$, that is the nilpotent-constrained radical of $G$. As $\mathrm{F}(X) \mathrm{F}(G), \mathrm{F}\left(Y_{1}\right) \mathrm{F}(G)$ are nilpotent subgroups of $C$ and $\mathrm{F}\left(Y_{1}\right) \leq \mathrm{N}_{C}(\mathrm{~F}(X))$ it follows from $([1])$ that $\mathrm{F}\left(Y_{1}\right) \mathrm{F}\left(\mathrm{N}_{C}(\mathrm{~F}(X))\right)$ is nilpotent.

On the other hand, as $X=\mathrm{F}(X) \mathrm{E}(X)$ and $\mathrm{E}(X) \leq \mathrm{E}\left(X \mathrm{~F}^{*}(G)\right)=\mathrm{E}(G)$ it follows that $\mathrm{N}_{C}(\mathrm{~F}(X))=\mathrm{N}_{C}(X)$. Moreover, $\mathrm{N}_{C}(X)=C \cap \mathrm{N}_{G}(X) \unlhd$ $\mathrm{N}_{G}(X)$, thus $\mathrm{F}\left(\mathrm{N}_{C}(X)\right) \leq \mathrm{F}\left(\mathrm{N}_{G}(X)\right) \leq C$, hence $\mathrm{F}\left(\mathrm{N}_{C}(X)\right)=\mathrm{F}\left(\mathrm{N}_{G}(X)\right)$ and $\mathrm{F}\left(Y_{1}\right) \mathrm{F}\left(\mathrm{N}_{G}(X)\right)$ is nilpotent. As $Y_{1} \mathrm{~F}^{*}(G) / \mathrm{E}(G)$ is nilpotent, it follows that $\mathrm{E}\left(Y_{1}\right) \leq \mathrm{E}(G)$. Therefore $Y \mathrm{~F}^{*}\left(\mathrm{~N}_{G}(X)\right)=Y_{1} \mathrm{~F}^{*}\left(\mathrm{~N}_{G}(X)\right)=$ $\mathrm{F}\left(Y_{1}\right) \mathrm{F}\left(\mathrm{N}_{G}(X)\right) \mathrm{E}(G)$ is quasinilpotent.

Corollary 7 Let $X \unlhd \unlhd X \mathrm{~F}^{*}(G)$, where $X \mathrm{~F}^{*}(G)$ is a quasinilpotent subgroup of $G$ and let $Y$ be a quasinilpotent injector of $\mathrm{N}_{G}(X)$. Then there exists a quasinilpotent injector $K$ of $G$ satisfying $K \cap \mathrm{N}_{G}(X)=Y$.

Proof. By Theorem 6, $Y \mathrm{~F}^{*}(G)$ is quasinilpotent. Let $K$ be a maximal quasinilpotent subgroup of $G$ containing $Y \mathrm{~F}^{*}(G)$, then $K$ is a quasinilpotent injector of $G$. Thus $K=\mathrm{E}(G) I$, where $I$ is a nilpotent injector of $\mathrm{C}_{G}(\mathrm{E}(G))$; hence $Y \leq K \cap \mathrm{N}_{G}(X)=\mathrm{E}(G)\left(I \cap \mathrm{N}_{G}(X)\right)$, that is quasinilpotent. Therefore $Y=K \cap \mathrm{N}_{G}(X)$.

Recall that, if $H \leq G$, it is said that $H$ controls its own $G$-fusion (briefly $H$ is c-closed in $G$ ), if any two elements of $H$, that are $G$-conjugate, are 
already $H$-conjugate. It is well known the Frobenius theorem, that states that in a finite group $G$, a Sylow $p$-subgroup of $G$ is c-closed in $G$ if and only if $G$ has a normal $p$-complement. Also, C. Sah proved, in $\pi$-separable groups, an analogous result for Hall $\pi$-subgroups. We will prove corresponding results for nilpotent injectors in nilpotent-constrained groups and for quasinilpotent injectors in finite groups.

Lemma 8 Let $H$ be c-closed in $G$. Then:

(i) $H \leq K \leq G$ implies that $H$ is c-closed in $K$.

(ii) If $K \leq H \leq G$ and $K$ is c-closed in $H$ then $K$ is c-closed in $G$.

(iii) If $K \leq H$ and $K \unlhd G$ then $H / K$ is c-closed in $G / K$.

(iv) If $N \unlhd G$ and $(|N|,|H|)=1$ then $H N / N$ is c-closed $G / N$.

Proof. See ([13], 2.2)

Theorem 9 Let $G$ be a nilpotent-constrained group and let I be a nilpotent injector of $G$. The following conditions are equivalent:

(i) $G$ is nilpotent.

(ii) I is c-closed in $G$.

(iii) $\mathrm{F}(G)$ is c-closed in $G$.

Proof. Clearly (i) implies (ii).

(ii) $\Rightarrow$ (iii) Let $p \in \pi(|I|)$. As $I$ is c-closed in $G$ it follows that $I_{p}$ is c-closed in $G$. Since $I_{p} \in \operatorname{Syl}_{p}\left(\mathrm{C}_{G}\left(\mathrm{O}_{p^{\prime}}(\mathrm{F}(G))\right)\right)$ by $([11], 1)$, then $I_{p}$ is cclosed in $C_{p}=\mathrm{C}_{G}\left(\mathrm{O}_{p^{\prime}}(\mathrm{F}(G))\right)$, thus $C_{p}$ is $p$-nilpotent $C_{p}=I_{p} \mathrm{O}_{p^{\prime}}\left(C_{p}\right)=$ $I_{p} \mathrm{Z}\left(\mathrm{O}_{p^{\prime}}(\mathrm{F}(G))\right)$, therefore $I_{p} \unlhd C_{p}$ and then $I_{p}=\mathrm{O}_{p}\left(C_{p}\right)=\mathrm{O}_{p}(G)$.

Hence $\mathrm{F}(G)=I$ and $\mathrm{F}(G)$ is c-closed in $G$.

(iii) $\Rightarrow$ (i) Suppose that there exists $p \in \pi(|G|) \backslash \pi(|\mathrm{F}(G)|)$. Let $P \in$ $\operatorname{Syl}_{p}(G)$, then $\mathrm{F}(G)$ is a Hall $p^{\prime}$-subgroup of $\mathrm{F}(G) P$ and $\mathrm{F}(G)$ is c-closed in $\mathrm{F}(G) P$. Then, by $([13], 1)$, we obtain that $P \unlhd \mathrm{F}(G) P$ so $P \leq \mathrm{C}_{G}(\mathrm{~F}(G)) \leq$ $\mathrm{F}(G)$, that is a contradiction. Consequently, $\pi(|\mathrm{F}(G)|)=\pi(|G|)$.

As $\mathrm{F}(G)$ is c-closed in $G$, it follows that $\mathrm{O}_{p^{\prime}}(\mathrm{F}(G))$ is c-closed in $G$, for every $p \in \pi(|G|)$. Take $P \in \operatorname{Syl}_{p}(G)$, then $\mathrm{O}_{p^{\prime}}(\mathrm{F}(G))$ is c-closed in $P \mathrm{O}_{p^{\prime}}(\mathrm{F}(G))$, thus $P \unlhd P \mathrm{O}_{p^{\prime}}(\mathrm{F}(G))$ by $([13], 1)$. Then $P \leq \mathrm{C}_{G}\left(\mathrm{O}_{p^{\prime}}(\mathrm{F}(G))\right)$ and by $([11], 1)$, we conclude that $G$ is nilpotent. 
Theorem 10 Let I be a quasinilpotent injector of $G$. The following conditions are equivalent:

(i) $G$ is quasinilpotent.

(ii) I is c-closed in $G$.

(iii) $\mathrm{F}^{*}(G)$ is c-closed in $G$.

Proof. Clearly (i) implies (ii).

(ii) $\Rightarrow$ (iii) We know that $I=\mathrm{E}(G) V$ where $V$ is a nilpotent injector of $\mathrm{C}_{G}(\mathrm{E}(G))$.

Since $V$ is c-closed in $\mathrm{C}_{G}(\mathrm{E}(G))$, by Theorem 9 , it follows that $\mathrm{C}_{G}(\mathrm{E}(G))$ is nilpotent. Therefore $\mathrm{C}_{G}(\mathrm{E}(G))=\mathrm{F}(G)$, and $I=\mathrm{E}(G) \mathrm{F}(G)=\mathrm{F}^{*}(G)$.

(iii) $\Rightarrow$ (i) By induction on order of $G$. Suppose that $Z=\mathrm{Z}(G) \neq$ 1. Then, by Lemma 8 (iii) and the inductive hypothesis, we obtain that $G / Z=\mathrm{F}^{*}(G / Z)=\mathrm{F}^{*}(G) / Z$ so $G=\mathrm{F}^{*}(G)$. Therefore, we can suppose that $Z=1$. Since $G=\mathrm{F}^{*}(G) \mathrm{C}_{G}(x)$, for every $x \in \mathrm{F}^{*}(G)$, we can conclude that $\mathrm{Z}(\mathrm{F}(G)) \leq \mathrm{Z}(G)=1$, thus $\mathrm{F}(G)=1$ and $\mathrm{F}^{*}(G)=\mathrm{E}(G)$.

Suppose that $\mathrm{E}(G) \leq L$, where $L$ is a maximal subgroup of $G$. By Lemma 1 (1) it follows that $\mathrm{E}(G)=\mathrm{E}(L)$, and as $\mathrm{F}(L) \leq \mathrm{C}_{G}(\mathrm{E}(G))=\mathrm{Z}(\mathrm{E}(G))=1$ we conclude, by induction, that $\mathrm{E}(G)=L$. Then $\mathrm{E}(G)$ is a maximal subgroup of $G$, so there exists a prime $p$ such that $|G / \mathrm{E}(G)|=p$.

Let $Q$ be a component of $G$. Since $G=\mathrm{E}(G) \mathrm{C}_{G}(x)$ for all $x \in \mathrm{E}(G)$, it follows that $\mathrm{Z}(Q)=1$. Therefore $\mathrm{E}(G)=Q_{1} \times \ldots \times Q_{r}$, where $Q_{1} \ldots, Q_{r}$ are the components of $G$ which are nonabelian simple groups. Also they are c-closed in $G$.

Let $i \in\{1, \ldots, r\} g \in G$ and let $\alpha_{g}$ be the inner automorphism of $G$ determined by $g$. Since $Q_{i} \unlhd G$, one has that the restriction $\alpha_{g \mid Q_{i}}$ is an automorphism of $Q_{i}$. Note that $\alpha_{g}(C)=C$ for any conjugacy class $C$ of $Q_{i}$; hence, by ([4], Theorem $\mathrm{C}$ ), there exists $z_{i} \in Q_{i}$ such that $\alpha_{g}(x)=x^{z_{i}}$ for every $x \in Q_{i}$. If $x \in E(G)$, then $x=x_{1} \ldots x_{r}, x_{i} \in Q_{i}, 1 \leq i \leq r$. Thus, $x^{g}=x_{1}^{g} \ldots x_{r}^{g}=x_{1}^{z_{1}} \ldots x_{r}^{z_{r}}=x_{1}{ }^{z} \ldots x_{r}{ }^{z}=x^{z}$, where $z=z_{1} \ldots z_{r} \in E(G)$. Therefore $\left.\alpha_{g}\right|_{\mathrm{E}(G)}$ is the inner automorphism of $\mathrm{E}(G)$ of $G$ determined by $z=z_{1} \ldots z_{r}$. It follows from ([7], X 13.1) that $G$ is quasinilpotent.

Corollary 11 If $G$ is a group then

$$
\mathrm{F}^{*}(G)=\bigcap_{\theta \in \operatorname{Irr}\left(\mathrm{F}^{*}(G)\right)} \mathrm{I}_{G}(\theta) .
$$


Proof. Work by induction on $|G|$. We know that

$$
\mathrm{F}^{*}(G) \leq \bigcap_{\theta \in \operatorname{Irr}\left(\mathrm{F}^{*}(G)\right)} \mathrm{I}_{G}(\theta)=N \unlhd G
$$

Suppose that $N<G$; since $\mathrm{F}^{*}(G)=\mathrm{F}^{*}(N)$, by induction, it follows that

$$
\mathrm{F}^{*}(G)=\mathrm{F}^{*}(N)=\bigcap_{\theta \in \operatorname{Irr}\left(\mathrm{F}^{*}(N)\right)} \mathrm{I}_{G}(\theta)=N .
$$

Therefore, we can suppose that $N=G$. Then $\mathrm{I}_{G}(\theta)=G$, for all $\theta \in$ $\operatorname{Irr}\left(\mathrm{F}^{*}(G)\right)$. Let $\operatorname{Irr}\left(\mathrm{F}^{*}(G)\right)=\left\{\theta_{1}, \theta_{2}, . ., \theta_{m}\right\}$. Suppose that $x, y \in \mathrm{F}^{*}(G)$ with $x^{g}=y$, for some $g \in G$, then

$$
\theta_{i}(x)=\theta_{i}^{g^{-1}}(x)=\theta_{i}\left(x^{g}\right)=\theta_{i}(y), 1 \leq i \leq m .
$$

Thus $\sum \theta_{i}(x) \theta_{i}\left(y^{-1}\right)=\sum \theta_{i}(y) \theta_{i}\left(y^{-1}\right) \neq 0$. Then $x$ and $y$ are conjugate in $\mathrm{F}^{*}(G)$ and, in consequence, $\mathrm{F}^{*}(G)$ is c-closed in $G$. Then, using Theorem 10 , it follows that $G=\mathrm{F}^{*}(G)$ as desired.

Corollary 12 If $G$ is a nilpotent-constrained group then

$$
\mathrm{F}(G)=\bigcap_{\theta \in \operatorname{Irr}(\mathrm{F}(G))} \mathrm{I}_{G}(\theta) .
$$

Proof. Since $G$ is a nilpotent-constrained group, we have $\mathrm{F}^{*}(G)=\mathrm{F}(G)$. Now apply the above result.

Corollary 13 Let $\mathfrak{F}$ be a Fitting class such that $\mathfrak{N} \subseteq \mathfrak{F} \subseteq \mathfrak{N}^{*}$ and let $G$ be an $\mathfrak{F}$-constrained group ( i.e. $\left.\mathrm{C}_{G}\left(G_{\mathfrak{F}}\right) \leq G_{\mathfrak{F}}\right)$. If $I \in \operatorname{Inj}_{\mathfrak{F}}(G)$, the following statements are equivalent:

(i) $G \in \mathfrak{F}$.

(ii) I is c-closed in $G$.

(iii) $G_{\mathfrak{F}}$ is c-closed in $G$.

Proof. Note that, as $G$ is an $\mathfrak{F}$-constrained group, by $([9], 2)$, we have $\mathrm{F}^{*}(G)=G_{\mathfrak{F}}$ and, by $([9], 8), \operatorname{Inj}_{\mathfrak{F}}(G)=\operatorname{Inj}_{\mathfrak{N}^{*}}(G)$. Now the result follows from Theorem 10. 


\section{Remarks.}

1. The last results suggest that, perhaps, one can obtain a general result for any Fitting class, but there exist Fitting classes of full characteristic and finite groups, do not belong to the corresponding Fitting class, but whose injectors are c-closed:

Consider $G=\mathrm{A}_{5}$ and $\mathfrak{F}=\mathfrak{S}$. If $S \in \operatorname{Syl}_{5}(G)$ then $\mathrm{N}_{G}(S) \cong \mathrm{D}_{10}$ is an $\mathfrak{F}$ injector of G. Moreover $\mathrm{N}_{G}(S)$ is c-closed in $\mathrm{A}_{5}$. Indeed, let $x \in \mathrm{N}_{G}(S) \backslash\{1\}$ and $g \in G$ such that $x^{g} \in \mathrm{N}_{G}(S)$.

If o $(x)=5$, then $\langle x\rangle=S$ and we obtain that $g \in \mathrm{N}_{G}(S)$.

If o $(x)=2$, then $\langle x\rangle,\left\langle x^{g}\right\rangle$ are Sylow 2-subgroups of $\mathrm{N}_{G}(S)$, thus there exists $h \in \mathrm{N}_{G}(S)$ such that $\left\{1, x^{g}\right\}=\left\langle x^{g}\right\rangle=\langle x\rangle^{h}=\left\{1, x^{h}\right\}$ and so $x^{g}=x^{h}$.

2. Even more, there exist Fitting classes of soluble groups with full characteristic and soluble groups, do not belong to the corresponding Fitting class, but whose injectors are c-closed:

If $G$ is a soluble group, we define an homomorphism $\mathrm{d}_{G}: G \longrightarrow \mathrm{GF}(5)^{*}$ as follows: let $M_{1}, M_{2}, \ldots, M_{r}$ the 5 -chief factors of a prefixed chief series of $G$. If $g \in G$ and $\mathrm{d}_{i}(g)$ denotes the determinant of the linear map which $g$ induces on $M_{i}$, then

$$
\mathrm{d}_{G}(g)=\prod_{i=1}^{r} \mathrm{~d}_{i}(g)
$$

The class $\mathfrak{F}=\left\{G \in \mathfrak{S} \mid \mathrm{d}_{G}(G)=1\right\}$ is a normal Fitting class ([3], IX 2.14). Let

$$
A=\left\langle\left(\begin{array}{ll}
2 & 0 \\
0 & 2
\end{array}\right),\left(\begin{array}{ll}
0 & 1 \\
1 & 0
\end{array}\right),\left(\begin{array}{ll}
0 & 3 \\
2 & 0
\end{array}\right)\right\rangle \leq G L(2,5) .
$$

Consider $A$ acting in the natural way on $\mathrm{C}_{5} \times \mathrm{C}_{5}$. Let $G$ be the semidirect product of $\mathrm{C}_{5} \times \mathrm{C}_{5}$ by $A$ :

$$
G=\left[\mathrm{C}_{5} \times \mathrm{C}_{5}\right]\left\langle\left(\begin{array}{cc}
2 & 0 \\
0 & 2
\end{array}\right),\left(\begin{array}{cc}
0 & 1 \\
1 & 0
\end{array}\right),\left(\begin{array}{ll}
0 & 3 \\
2 & 0
\end{array}\right)\right\rangle
$$

and let

$$
S=\left[\mathrm{C}_{5} \times \mathrm{C}_{5}\right]\left\langle\left(\begin{array}{cc}
3 & 0 \\
0 & 2
\end{array}\right),\left(\begin{array}{ll}
0 & 2 \\
2 & 0
\end{array}\right)\right\rangle
$$

Observe that $|G|=2^{4} \cdot 5^{2}=400$ and $|S|=2^{3} \cdot 5^{2}$.

We will see that $S$ is c-closed in $G$.

We have that $G=S\left\langle\left(\begin{array}{ll}2 & 0 \\ 0 & 2\end{array}\right)\right\rangle$ and if $S_{2}=\left\langle\left(\begin{array}{ll}3 & 0 \\ 0 & 2\end{array}\right),\left(\begin{array}{ll}0 & 2 \\ 2 & 0\end{array}\right)\right\rangle \in$ $\operatorname{Syl}_{2}(S)$ then $\left\langle\left(\begin{array}{ll}2 & 0 \\ 0 & 2\end{array}\right)\right\rangle \leq \mathrm{C}_{G}\left(S_{2}\right)$. 
Hence, if $x \in S_{2}$ y $g \in G$, we have $g=c s$, where $c \in\left\langle\left(\begin{array}{ll}2 & 0 \\ 0 & 2\end{array}\right)\right\rangle$, so $x^{g}=x^{c s}=x^{s}$. Therefore $G=\mathrm{C}_{G}(x) S$.

Let $x \in S$. Since $S$ does not have composed order elements, $x$ is a 2-element or a 5-element.

If $x$ is a 2-element then $x=y^{s}$ where $y \in S_{2}$ and $s \in S$.

Hence $\mathrm{C}_{G}(x) S=\mathrm{C}_{G}\left(y^{s}\right) S=\left(\mathrm{C}_{G}(y)\right)^{s} S=\left(\mathrm{C}_{G}(y) S\right)^{s}=G$. Thus, if $g \in G$, it follows that $g=l s$, where $l \in \mathrm{C}_{G}(x), s \in S$. Then $x^{g}=x^{l s}=x^{s}$.

If $x$ is a 5-element, then $x \in \mathrm{C}_{5} \times \mathrm{C}_{5}$. We will see that $G=\mathrm{C}_{G}(x) S$. It is enough to prove that there exists $g \in G \backslash S$ such that $g \in \mathrm{C}_{G}(x)$.

If $H \leq G$ write $H^{*}=H \backslash\{1\}$. Then

$$
\left(\mathrm{C}_{5} \times \mathrm{C}_{5}\right)^{*}=\left\langle h_{1}\right\rangle^{*} \cup\left\langle h_{2}\right\rangle^{*} \cup\left\langle h_{3}\right\rangle^{*} \cup\left\langle h_{4}\right\rangle^{*} \cup\left\langle h_{5}\right\rangle^{*} \cup\left\langle h_{6}\right\rangle^{*}
$$

where $h_{1}=(1,0), h_{2}=(0,1), h_{3}=(1,1), h_{4}=(2,1), h_{5}=(1,2), h_{6}=$ $(4,1)$.

Notice that if $h \in\left\langle h_{i}\right\rangle$, then $\mathrm{C}_{G}(h)=\mathrm{C}_{G}\left(h_{i}\right) 1 \leq i \leq 6$ and it is enough to show that $G=\mathrm{C}_{G}\left(h_{i}\right) S, 1 \leq i \leq 6$.

We have,

$$
\begin{aligned}
& \left(\begin{array}{ll}
1 & 0 \\
0 & 4
\end{array}\right) \in \mathrm{C}_{G}\left(h_{1}\right) \backslash S,\left(\begin{array}{ll}
4 & 0 \\
0 & 1
\end{array}\right) \in \mathrm{C}_{G}\left(h_{2}\right) \backslash S,\left(\begin{array}{ll}
0 & 1 \\
1 & 0
\end{array}\right) \in \mathrm{C}_{G}\left(h_{3}\right) \backslash S, \\
& \left(\begin{array}{ll}
0 & 2 \\
3 & 0
\end{array}\right) \in \mathrm{C}_{G}\left(h_{4}\right) \backslash S,\left(\begin{array}{ll}
0 & 3 \\
2 & 0
\end{array}\right) \in \mathrm{C}_{G}\left(h_{5}\right) \backslash S,\left(\begin{array}{ll}
0 & 4 \\
4 & 0
\end{array}\right) \in \mathrm{C}_{G}\left(h_{6}\right) \backslash S,
\end{aligned}
$$

Hence $G=\mathrm{C}_{G}(x) S$. Therefore, if $g \in G, g=c s$, where $c \in \mathrm{C}_{G}(x)$ and $s \in S$. Then $x^{g}=x^{c s}=x^{s}$.Thus, $S$ is c-closed in $G$.

Now consider the chief series of $G$ :

$$
\begin{aligned}
1 \unlhd \mathrm{C}_{5} \times \mathrm{C}_{5} & \unlhd\left[\mathrm{C}_{5} \times \mathrm{C}_{5}\right]\left\langle\left(\begin{array}{ll}
4 & 0 \\
0 & 4
\end{array}\right)\right\rangle \\
& \unlhd\left[\mathrm{C}_{5} \times \mathrm{C}_{5}\right]\left\langle\left(\begin{array}{ll}
4 & 0 \\
0 & 4
\end{array}\right),\left(\begin{array}{ll}
0 & 2 \\
2 & 0
\end{array}\right)\right\rangle \unlhd S \unlhd G .
\end{aligned}
$$

The only 5-chief factor of this series is $\mathrm{C}_{5} \times \mathrm{C}_{5}$.

Notice that $G \notin \mathfrak{F}$ since $\operatorname{det}\left(\begin{array}{ll}2 & 0 \\ 0 & 2\end{array}\right)=4 \neq 1$.

The part of the above series from 1 to $S$ is a chief series of $S$ and the only 5-chief factor of this series is $\mathrm{C}_{5} \times \mathrm{C}_{5}$. 
Since $\operatorname{det}\left(\begin{array}{ll}3 & 0 \\ 0 & 2\end{array}\right)=1$ and $\operatorname{det}\left(\begin{array}{ll}0 & 2 \\ 2 & 0\end{array}\right)=1$, it follows that $S \in \mathfrak{F}$, then $S \in \operatorname{Iny}_{\mathfrak{F}}(G)$ is c-closed in $G$, but $G \notin \mathfrak{F}$.

3. It is said that a subgroup $H$ in a group $G$ has property CR (Character Restriction) if every ordinary irreducible character $\theta \in \operatorname{Irr}(H)$ is the restriction $\chi_{H}$ of some $\chi \in \operatorname{Irr}(G)$. It is well known that if $H$ satisfies CR property in $G$ then $H$ is c-closed in $G$.

A number of authors have shown that property CR, together with suitable additional hypothesis on $H$ and $G$, does imply the existence of a normal complement for $H$. For instance Hawkes and Humphreys ([5]) prove that CR yields a normal complement if $G$ is solvable and $H$ is an $\mathfrak{F}$-projector for $G$, where $\mathfrak{F}$ is any saturated formation. The last example shows that the corresponding result for Fitting classes and injectors satisfying property CR, does not work.

\section{References}

[1] Bender, H.: Nilpotent $\pi$-subgroups behaving like $p$-subgroups. Proceedings of the Rutgers group theory year, 1983-1984 1 (1984), 119-125, Cambridge Univ. Press, Cambridge, 1985.

[2] Bender, H.: On the normal $p$-structure of a finite group and related topics. Hokkaido Math. J. 7 (1978), 271-288.

[3] Doerk, K. And Hawkes, T.: Finite Soluble Groups. Walter de Gruyter, Berlin-New York, 1992.

[4] Feit, W. And Seitz, G.M.: On finite rational matrix groups and related topics. Illinois J. Math. 33 (1988), 103-131.

[5] Hawkes, T. O. and Humphreys, J. F.: A character Theoretic criterion for the existence of normal complements to subgroups of finite groups. J. Algebra 94 (1985), 382-387.

[6] Huppert, B.: Endliche Gruppen I. Die Grundlehren der Mathematischen Wissenschaften, Band 134. Springer-Verlag, Berlin-New York, 1967.

[7] Huppert, B. and Blackburn, N.: Finite Groups III. Springer-Verlag, Berlin-Heidelberg-New York, 1982.

[8] Iranzo, M.J., Medina, J. And Pérez-Monasor, F.: Product of nilpotent subgroups. Arch. Math. (Basel) 74 (2000), 246-252.

[9] Iranzo, M.J. And PÉrez-Monasor, F.: $\mathfrak{F}$-constraint with respect to a Fitting class. Arch. Math. (Basel) 46 (1986), 205-210.

[10] Kurzweil, H.: Endliche Gruppen Eine Einfhrung in die Theorie der endlichen Gruppen. Hochschultext, Springer-Verlag, Berlin-New York, 1977.

[11] Mann, A.: Injectors and normal subgroups of finite groups. Israel J. Math. 9 (1971), 554-558. 
[12] Navarro, G. And Sanus, L.: A partition of characters associated to nilpotent subgroups. Israel J. Math. 114 (1999), 359-380.

[13] SAH, C.: Existence of normal complements and extension of characters in finite groups. Illinois J. Math 6 (1962), 282-291.

Recibido: 25 de mayo de 2001

M. J. Iranzo

Departamento de Álgebra

Universidad De Valencia

C/ Dr. Moliner, 50

46100 Burjasot, Valencia, Spain

M. Jesus. Iranzo@uv. es

J. Medina

Departamento de Matemática Aplicada

Universidad Politécnica de Cartagena

P. Alfonso XIII, 52

30203 Cartagena, Murcia, Spain

juan.medina@upct.es

F. Pérez-Monasor ${ }^{1}$

Departamento de Álgebra

Universidad De Valencia

C/ Dr. Moliner, 50

46100 Burjasot, Valencia, Spain

F.Perez.Monasor@uv.es

\footnotetext{
${ }^{1}$ Supported in part by DGICYT, PB97-0674-C02.
} 